\title{
Cenni riassuntivi sulla teoria dei funzionali analitici
}

L. FANTAPPIÉ (Palermo).

In questo lavoro intendo riassumere per sommi capi i risultati da me conseguiti negli ultimi quattro anni nello studio degli operatori funzionali. Fin dall'inizio del 1925, seguendo l'indirizzo delle suggestive teorie sviluppate dal Prof. Volterra, mi occupai infatti dell'estensione al campo complesso della teoria dei „funzionali“, o "funzioni di linee“, fino allora trattata principalmente nel campo reale (quando si eccettuino i lavori del Prof. Pincherle sulle "operazioni distributive").

L'introduzione dell'immaginario, come già tante altre volte nello sviluppo delle teorie analitiche, rende possibile, anche in queste considerazioni, la completa coordinazione in un insieme armonico di tutte le principali proprietà dei funzionali, che effettivamente s'incontrano nell'analisi; cioè di quella classe particolare di funzionali, definiti per le funzioni analitiche, ma spesso prolungabili alle funzioni anche soltanto integrabili, o-derivabili, etc., che io chiamo "funzionali analitici".

Con questo riassunto, spero di potere interessare altri allo studio di questi "funzionali analitici“, offrendo insieme un mezzo di orientamento per la lettura di una memoria, ora in corso di stampa presso la R. Accademia dei Lincei, in cui viene completamente sviluppata la teoria generale di detti operatori.

INTORNO DI UNA FUNZIONE E FUNZIONALI ANALITICI LOCALMENTE.

Consideriamo il caso più semplice di un numero $f$ che dipenda da una sola funzione analitica e monodroma $y(t)$,

$$
f=F[y(t)]
$$


Si dirà che $f$ è un funzionale della funzione $y(t)$, definito in un certo campo funzionale $H$ di variabilità della $y(t)$, funzione variabile indipendente. Se la $y$ contiene, pure analiticamente, un parametro $\alpha$, se cioè $y=y(t, \alpha)$, per ogni valore di $\alpha$ avremo una particolare funzione di $t$, che, al variare del parametro $\alpha$, descriverà, nello spazio funzionale di tutte le funzioni analitiche, (punti di questo spazio) un insieme parziale, che chiameremo "linea analitica" dello spazio funzionale. Applicando alla funzione $y=y(t, c)$, considerata come funzione della sola $t$, il funzionale $F$, il che si indicherà col simbolo $F_{t}$, avremo per ogni valore del parametro $\alpha$ una funzione di $t$, e quindi un valore del funzionale, valore che dunque risulterà una funzione

(2)

$$
f(\alpha)=F_{t}[y(t, \alpha)]
$$

di $\alpha$, definita per tutti quei valori del parametro, che individuano funzioni della linea analitica, contenute nel campo $H$ di definizione del funzionale $F$.

Per definire con precisione i funzionali analitici, oggetto del nostro studio, premettiamo che chiameremo intorno $(r, \sigma)$ di una funzione analitica e monodroma $y_{0}(t)$ (nello spazio funzionale) l'insieme di tutte quelle funzioni analitiche e monodrome $y(t)$ che sono olomorfe in una regione $A(r)$ della sfera complessa, costituita da tutti i punti che hanno una "distanza sferica“ maggiore o uguale a $r$ dai punti non regolari della $y_{0}(t)$, e che, in questa regione, soddisfano alla diseguaglianza

$$
\left|y(t)-y_{0}(t)\right|<\sigma
$$

Al crescere di $\sigma$, l'intorno $(r, \sigma)$ cresce pure, nel senso che viene a contenere sempre nuove funzioni, finchè, per $\sigma=\infty$, avremo tutto l'insieme delle funzioni $y(t)$ che sono semplicemente olomorfe nella regione $A(r)$, insieme che indicheremo più brevemente come intorno $(r)$, invece che $(r, \infty)$.

Ciò premesso si dirà che un funzionale $F$, univocamente definito in un intorno $(r, \sigma)$ di una funzione $y_{0}(t)$ è ivi regolare, quando accada che, scelta comunque una linea analitica $y=y(t, \alpha)$, che abbia una parte interna a questo intorno, costituita dalle funzioni corrispondenti ai valori di $\alpha$ di una certa regione $T$, la funzione

$$
f(\alpha)=F_{t}[y(t, \alpha)]
$$

sia sempre olomorfa nella regione $T$, se la $y(t, \alpha)$ é pure olomorfa, non solo per $t$ in $A(r)$, ma anche per $\omega$ in $T$ (è cioè olomorfa rispetto ad ambedue le variabili $t$ e $c$ ).

Si dirà poi che un funzionale $F$, univocamente definito in un campo funzionale $H$, è ivi analitico localmente, se esso è regolare nell'intorno di ogni funzione del campo. In questo caso la funzione $f(\alpha)=F_{t}[y(t, \alpha)]$, che nasce dall'applicazione di $F$ a una funzione $y$, variabile su una linea analitica, è pure sempre analitica; in altri termini possiamo dire che la proprietà caratteristica dei funzionali analitici localmente è quella di conservare l'analiticità rapporto ai parametri. Questa proprietà sembra doversi considerare di importanza assolutamente prevalente sopra ogni altra, anche per il fatto che tutte le dipendenze funzionali che già si sono incontrate nei vari rami dell'analisi, rivestono per l'appunto questo carattere, come per esempio quella degli integrali di una equazione a derivate parziali dalle funzioni iniziali, degli integrali di una equazione differenziale lineare dai coefficienti dell'equazione stessa, e cosi pure hanno questo carattere i funzionali studiati dal Prof. Volterra e anche quelli più generali (definiti nel campo di tutte le funzioni continue in un intervallo) trattati in una memoria del Prof. Fréchet (Ann. de l'École normale sup., 1910).

\section{CONNESSIONE DEI CAMPI FUNZIONALI.}

Analogamente a quanto si fa per le regioni in cui sono definite delle funzioni monogene, possiamo ora cercare di stabilire il concetto di connessione di un campo funzionale $H$, in cui è definito univocamente un funzionale $F$, analitico localmente. Se si può trovare una linea analitica $y=y(t, \alpha)$ tale che, facendo variare $\alpha$ su un conveniente cammino da $\alpha_{1}$ a $\alpha_{2}$ la funzione corrispondente sulla linea si mantiene sempre, insieme con un suo intorno, nel campo funzionale $H$, variando dalla funzione iniziale $y_{1}(t)=y\left(t, \alpha_{1}\right)$ alla funzione finale $y_{2}(t)=y\left(t, \alpha_{2}\right)$, diremo che è possibile passare con continuità, entro $H$, dalla funzione $y_{1}(t)$ alla funzione $y_{2}(t)$. Se da questa è possibile passare con continuità entro $H$ a una terza funzione $y_{3}(t)$, scegliendo un' altra conveniente linea analitica, diremo anche che si può passare. con continuità entro $H$ da $y_{1}$ a $y_{3}$, e così via. 
Date due qualunque funzioni del campo funzionale $H$, diremo allora che il campo stesso è connesso, se si può sempre passare con continuità entro $H$ dall'una all'altra di esse. In questo caso, un funzionale $F$, che sia univocamente definito nel campo $H$ $\mathrm{e}$ analitico localmente, gode di una proprietà particolarmente interessante. Basta infatti conoscere i valori assunti dal funzionale per tutte le funzioni $y(t)$ di un intorno $(r, \sigma)$ comunque piccolo di una data funzione $y_{o}(t)$ (un "elemento" del funzionale $F$, di centro $y_{o}(t)$, come diremo più brevemente) per conoscere il valore del funzionale stesso per qualunque altra funzione $y(t)$ del suo campo di definizione. Nel caso più semplice che basti una sola linea analitica $y=y(t, \alpha)$ per passare da $y_{0}(t)=y\left(t, \alpha_{o}\right)$ a $y_{1}(t)=y\left(t, \alpha_{1}\right)$, ciò si vede subito osservando che la funzione $f(\alpha)=F_{t}[y(t, \alpha)]$, olomorfa su tutto il cammino $L$, congiungente $\alpha_{o}$ con $\alpha_{1}$, risulta nota per i valori di $\alpha$ in un intorno di $\alpha_{o}$, a cui corrispondono funzioni interne all'intorno $(r, \sigma)$ di $y_{o}(t)$; prolungando analiticamente la $f(\alpha)$ lungo $L$ fino al valore $\alpha_{1}$ si avrà appunto $f\left(\alpha_{1}\right)=F\left[y\left(t, \alpha_{1}\right)\right]=F\left[y_{1}(t)\right]$.

FUNZIONALI ANALITICI IN SENSO STRETTO E LORO CAMPO NATURALE DI ESISTENZA.

La proprietà precedente dei funzionali analitici localmente in un campo connesso, che fa perfetto riscontro alla analoga proprietà delle funzioni analitiche (secondo Weierstrass) di essere completamente individuate dai valori assunti nell'intorno, comunque piccolo, di un punto regolare, suggerisce naturalmente di spingere ancora più avanti l'analogia tra le funzioni analitiche e i funzionali analitici.

Presa una funzione $y_{o}(t)$ del campo, in un intorno della quale il funzionale $F$ è certo regolare, possiamo infatti cercare qualche espressione che ci dia $F$ in questo intorno, ma conservi un significato anche per le funzioni di un intorno più ampio, per cui $F$ non era inizialmente definito. Per queste funzioni, esterne al campo iniziale $H$, potremo allora prendere per valore del funzionale il valore dato dall'espressione stessa, semprechè nell'intorno più ampio il funzionale così definito si mantenga regolare. In questo caso diremo anche che il funzionale $F$ è stato prolungato analiticamente a tutto questo intorno più ampio. Così seguitando potremo immaginare di eseguire tutti i possibili prolungamenti analitici del funzionale iniziale $F$ a successivi intorni, costruendo altrettanti „elementi ${ }^{\prime \prime}$ di un nuovo funzionale $\bar{F}$, che coincide con $F$ nel campo iniziale, e che è pure analitico localmente in un campo funzionale che, per la costruzione stessa, è ancora connesso. Questo campo funzionale ampliato si dirà il campo naturale di esistenza del funzionale $\bar{F}$, che si chiamerà anche analitico in senso stretto. In altri termini possiamo anche dire che un funzionale analitico in senso stretto non è altro che un funzionale analitico localmente, definito in un campo funzionale connesso (campo naturale di esistenza), non ulteriormente ampliabile con prolungamenti analitici.

Se invece il funzionale $F$, analitico localmente, era definito inizialmente in un campo funzionale non connesso, non è in generale possibile il suo prolungamento analitico in un medesimo funzionale $\bar{F}$, analitico in senso stretto. (Questa distinzione dei funzionali analitici in senso stretto da quelli analitici localmente è dovuta ad alcune osservazioni dei Proff. Severi e Bagnera sui miei lavori del 1925 e 1926, ancora imprecisi al riguardo).

Naturalmente, come avviene per le funzioni analitiche, può benissimo accadere che un funzionale analitico in senso stretto risulti polidromo, cioè due elementi diversi diano per una stessa funzione valori diversi del funzionale.

\section{FUNZIONALI LINEARI ANALITICI LOCALMENTE.}

Premesse queste nozioni generali sui funzionali analitici, andiamo ora ad esaminare una classe particolarmente importante di questi funzionali, e cioè i cosidetti funzionali lineari, caratterizzati dal fatto di godere della proprietà distributiva rispetto alla somma:

$$
F\left[y_{1}(t)+y_{2}(t)\right]=F\left[y_{1}(t)\right]+F\left[y_{2}(t)\right] .
$$

Lo studio di tutti i possibili funzionali lineari analitici localmente e univocamente definiti si esaurisce nel modo più semplice, introducendo, per ogni tale funzionale $F$, una funzione analitica caratteristica, definita da

$$
F_{t}\left[\frac{1}{t-\alpha}\right]=v(\alpha)
$$

che si ottiene applicando il funzionale stesso alle funzioni della Studia Mathematica. 
particolare linea analitica $y=\frac{1}{t-\alpha}$, che risultino interne al campo funzionale $H$, in cui $F$ è univocamente definito. L'insieme dei valori di $\alpha$, che individuano queste funzioni del campo $H$, risulta una regione $B$ della sfera complessa, che può anche non essere connessa (nel qual caso $H$ è pure un campo funzionale non connesso) in cui è definita la funzione monogena $v(\alpha)$, che si dice la funzione indicatrice del funzionale $F$. L'insieme complementare di $B$ sulla sfera complessa è invece un insieme chiuso $A$ (in cui l'indicatrice non è definita); il campo funzionale $H$ risulta poi dato, con alcune ipotesi supplementari (campo funzionale "lineare $\left.{ }^{6 t}\right)$ da tutte le funzioni $y(t)$ olomorfe nei punti di $A$.

L'importanza della funzione indicatrice di un funzionale lineare $F$ consiste principalmente in questo, che non solo, per la formula precedente, ogni tale funzionale individua perfettamente la sua funzione indicatrice, ma anche, viceversa, nota la funzione indicatrice $v(\alpha)$, è sempre possibile determinare il valore del funzionale lineare $F$ per qualunque funzione del suo campo di definizione.

Dimostrata infatti, sotto certe ipotesi, la derivabilità rapporto a un parametro sotto il segno di funzionale lineare, cioè

$$
\frac{d^{n}}{d \alpha^{n}} F_{t}[y(t, \alpha)]=F_{t}\left[\frac{\partial^{n}}{\partial \alpha^{n}} y(t, \alpha)\right]
$$

e l'applicabilità termine a termine a una serie, cioè

$$
F\left[\sum_{1}^{\infty} y^{n}(t)\right]=\sum_{1}^{\infty} F\left[\dot{y}_{n}(t)\right]
$$

si trova che, indicando con $v(\alpha)$ l'indicatrice del funzionale lineare $F$, il valore di $F$ per una qualunque funzione $y(t)$ del suo campo di definizione (olomorfa nei punti dellinsieme chiuso $A$ ) è dato dalla formula integrale:

$$
F[y(t)]=\frac{1}{2 \pi i} \int_{C} v(t) y(t) d t
$$

in cui $C$ è una curva chiusa della sfera complessa, che racchiude nell'interno tutti i punti non regolari della funzione variabile indipendente $y(t)$, in cui $y(t)$ non è in generale definita (ivi compreso l' $\infty$, se, pur essendo la $y(t)$ regolare all' $\infty$, è però $y(\infty) \neq 0$ ), ma lascia all'esterno tutti i punti dell'insieme chiuso
A, in cui l'indicatrice non è definita (curva che si dirà separatrice di questi due insiemi, e che, particolarmente nel caso che la regione $B$ non sia connessa, può anche essere costituita da più curve chiuse parziali).

E'appunto nella precisazione di questa curva separatrice come curva di integrazione il punto essenziale della formula (8).

La formula integrale (8), che io detti nell'aprile del 1925, e che è stata ritrovata per altra via dal Prof. Flamant coi metodi dell'analisi generale del Prof. Fréchet, ci fornisce dunque la più generale espressione possibile di tutti i funzionali lineari analitici localmente. Questa espressione integrale si indicherà anche col nome di prodotto funzionale delle due funzioni $v(t)$ e $y(t)$, e col simbolo

$$
v(t) y\left({ }_{*}^{*}\right)=\frac{1}{2 \pi i} \int_{C} v(t) y(t) d t
$$

in cui l'asterisco in alto sopra la $y$ serve ad indicare che la curva separatrice $C$ deve contenere nell'interno i punti in cui non è definita la $y$, e lasciare all'esterno quelli in cui non è definita la $v$, che porta l'asterisco in basso. Avremo naturalmente

$$
y(t) v(t)=\frac{1}{2 \pi i} \int_{-C} v(t) y(t) d t=-v(t) y(\stackrel{*}{*}),
$$

da cui il nome più preciso di prodotto funzionale emisimmetrico, dato a questa espressione. Il prodotto funzionale di due funzioni deve la sua importanza al fatto che costituisce l'operazione funzionale fondamentale, mediante la quale si possono costruire, come vedremo in seguito, tutti i funzionali analitici, anche non lineari.

FUNZIONALI LINEARI ANALITICI IN SENSO STRETTO.

Se la regione $B$, in cui è definita la funzione $v(\alpha)$, indicatrice di un funzionale lineare $F$, analitico localmente, è connessa, anche il campo funzionale $H$ in cui $F$ è definito, risulta connesso e se la $v(\alpha)$, monogena in $B$, è prolungabile analiticamente in una funzione $\bar{v}(\alpha)$, monodrom a su tutta la sfera complessa (cioè definita in una regione connessa $\bar{B}$, contenente $B$, ma non ricoprentesi più volte), anche il funzionale $F$, analitico localmente, è prolungabile analiticamente in un funzionale $\bar{F}$, 
analitico in senso stretto, definito univocamente dalla formula

$$
\bar{F}[y t)]=\frac{1}{2 \pi i} \int_{C} \bar{v}(t) y(t) d t=\bar{v}(t) y(\stackrel{*}{*})
$$

in tutto il campo funzionale connesso $\vec{H}$, (campo naturale di esistenza) dato dalle funzioni $y(t)$, olomorfe nei punti non regolari di $\bar{v}(t)$.

Se invece la $v(\alpha)$, definita nella regione connessa $B$, è prolungabile analiticamente in una funzione polidroma, il funzionale lineare $F$, che l'ammette per indicatrice è ancora prolungabile analiticamente in un funzionale $\bar{F}$, analitico in senso stretto, ma questo funzionale $\bar{F}$ risulta polidromo. A differenza di quanto accade per le funzioni analitiche, vediamo dunque che già tra $\mathrm{i}$ funzionali analitici line ari possono aversi esempi di funzionali polidromi.

Uno di questi esempi ci è dato dall'espressione

$$
F[y(t)]=\int_{L} k(t) y(t) d t
$$

(in cui $L$ è un cammino fisso e aperto della sfera complessa), espressione che è un funzionale lineare, analitico localmente e univocamente definito per tutte le funzioni $y(t)$, olomorfe su $L$ (linea a cui si riduce in questo caso l'insieme chiuso $A$ ). I prolungamenti analitici di questo funzionale si hanno considerando il cammino $L$ d'integrazione, non più fisso, ma variabile con continuità al variare continuo della funzione $y(t)$, variabile indipendente. L'importanza di questo funzionale $F$, prolungabile in un funzionale polidromo $\bar{F}$, consiste in ciò, che la sua definizione può estendersi nel modo piu naturale, colla stessa formula (12), dalle funzioni analitiche olomorfe su $L$, a tutte le funzioni, anche non analitiche, ma semplicemente integrabili su $L$.

\section{FUNZIONALI LINEARI MISTI (OPERAZIONI DISTRIBUTIVE).}

Finora abbiamo considerato i funzionali analitici lineari dipendenti solo da una funzione $y(t)$, che chiameremo anche funzionali puri, per distinguerli da quelli che, dipendendo contemporaneamente da funzioni e da numeri, si diranno funzionali misti. Il caso più semplice di funzionali misti si ha quando un numero $f$ dipende da una sola funzione $y(t)$ e da un solo parametro $z$

$$
f=F[y(t) ; z]
$$

Questa dipendenza tiene contemporaneamente della natura del funzionale, in quanto riguarda la funzione $y(t)$ variabile indipendente, e della natura della funzione, in quanto riguarda il numero $z$, pure variabile indipendente. Fissata la sola funzione $y(t), f$ resterà una funzione ordinaria di $z$, che supporremo pure sempre analitica; possiamo quindi anche dire che un funzionale misto (13) fa corrispondere ad ogni funzione $y(t)$, variabile indipendente, un'altra funzione $f(z)$

$$
f(z)=F[y(t) ; z] \text {. }
$$

Se invece fissiamo il parametro $z$ si ottiene un funzionale puro della $y(t)$, e nel caso che questo sia lineare e definito per $y(t)$ uguale a un polinomio qualunque, avremo quella vasta classe di funzionali, che il Prof. Pincherle ha cosi profondamente studiato nelle sue opere, col nome di operazioni funzionali distributive.

La teoria dei funzionali lineari misti si può però sviluppare anche fissando volta per volta il valore del parametro $z$, e applicando poi i risultati dei funzionali lineari puri. Così, per esempio, si dirà funzione indicatrice del funzionale lineare misto $F$, la funzione $v(z, \alpha)$ del parametro $z$, e dell' indice $\alpha$, definita dalla formula

$$
v(z, \alpha)=F_{t}\left[\frac{1}{t-\alpha} ; z\right]
$$

la quale è completamente individuata dal funzionale lineare $F$, e lo individua a sua volta, mediante la formula fondamentale (8), che in questo caso diventa

$$
\text { (16) } F[y(t) ; z]=v(z, t) y(t)=\frac{1}{2 \pi i} \int_{C} v(z, t) y(t) d t=f(z) \text {. }
$$

Da questa formula integrale si ricava anzi nel modo più facile un teorema della massima generalità sulla posizione delle singolarità della funzione $f(z)$, che viene a corrispondere per il funzionale lineare misto $F$, alla funzione $y(t)$, variabile indipendente.

Tenendo presente infatti la proprietà essenziale della curva $C$ d'integrazione di essere una curva separatrice, contenente 
nell'interno i punti singolari della $y(t)$, e lasciante all'esterno quelli della $v(z, t)$ (pensata come funzione della sola $t$ ) avremo che questa espressione perderà significato, oltre che per i valori $\bar{z}$ di $z$, per cui la $v \overline{(z}, t)$ stessa non è definita, (per nessun valore di $t$ ), solo per quei valori di $z$, per cui qualche punto $\bar{t}$, singolare per la $v(z, t)$ viene a coincidere con qualche punto singolare della $y(t)$, nel qual caso non esiste più una curva separatrice su cui eseguire la integrazione. Essendo la $f(z)$ regolare per ogni altro valore di $z$, i suoi punti singolari potranno dunque trovarsi solo tra i valori precedenti di $z$, i quali si ottengono tutti dai valori singolari delle funzioni $v\left(z, t_{r}\right)$ della $z$, che si hanno dalla indicatrice, quando al posto dell'indice si sostituiscano i valori $t_{r}$, singolari per la funzione $y(t)$. Note dunque le singolarità della funzione $y(t)$, variabile indipendente, e la funzione indicatrice $v(z, t)$, si determinano immediatamente, con questo teorema generale, le posizioni delle eventuali singolarità della funzione $f(z)$, dipendente linearemente dalla $y(t)$ (da ciò il nome di indicatrice dei punti singolari, dato alla funzione $v(z, t)$ nella prima nota dei Lincei del 1925).

E' da osservare poi che non solo a ogni funzionale lineare (puro o misto) corrisponde la rispettiva funzione indicatrice, ma anche, viceversa, presa ad arbitrio una funzione analitica $v(\alpha)$ - $v(z, \alpha)$, esiste sempre un funzionale lineare $F[y(t)]$ (puro) o $F[y(t) ; z]$ (misto) rispettivamente, che ammette questa funzione per indicatrice; bastando prendere per definizione di questi funzionali la formula (8) o (16). Se quindi prendiamo una funzione $v(z, \alpha)$ singolare per $\alpha=\infty$, qualunque sia $z$, il funzionale lineare misto $F[y(t) ; z]$, che l'ammette per indicatrice, non sarà definito per $y(t)$ uguale a un polinomio, essendo in questo caso anche la $y(t)$ singolare nello stesso punto $\infty$. Questi funzionali lineari misti, che hanno l'indicatrice singolare all' $\infty$, costituiscono dunque una classe di operazioni distributive, che non rientra tra quelle studiate dal Prof. Pincherle, date da espressioni del tipo

$$
F[y(t) ; z]=\sum_{0}^{\infty} \frac{\alpha_{n}(z)}{n !} y^{(n)}(z),
$$

le quali invece sono certo definite per $y$ uguale a un polinomio.

\section{FUNZIONALI LINEARI NORMALI.}

Come caso particolare del teorema sulle singolarità di una funzione $f(z)$ che dipende linearmente da un'altra $y(t)$, si ritrova il ben noto teorema di Hadamard sulle serie di potenze. Data infatti una serie di potenze

$$
k(z)=\sum_{0}^{\infty} k_{n} z^{n}
$$

che rappresenta una funzione singolare nei punti $z=\alpha_{r}$, se consideriamo il funzionale lineare misto $F[y(t) ; z]$ che ha per indicatrice la funzione

$$
v(z, \alpha)=-\frac{1}{\alpha} k\left(\frac{z}{\alpha}\right)
$$

troviamo che questo funzionale fa corrispondere a ogni potenza $y=t^{n}$, la potenza stessa moltiplicata per un fattore costante $k_{n}$, cioè

$$
F\left[t^{n} ; z\right]=k_{n} z^{n}
$$

I funzionali lineari misti, che godono della proprietà espressa dalla (20), seguendo la denominazione del Prof. Pincherle, saranno detti funzionali normali. Essi sono caratterizzati dal fatto di avere per indicatrice una funzione del tipo (19), cioè una funzione del quoziente del parametro per l'indice, divisa ancora per l'indice.

Applicando il funzionale $F$, che ha l'indicatrice (19), a una funzione $y(t)$, singolare per $t=\beta_{s}$, rappresentata da un'altra serie di potenze

$$
y(t)=\sum_{0}^{\infty} a_{n} t^{n}
$$

avremo per risultato una funzione $f(z)$, data dalla serie di potenze

$$
f(z)=F[y(t) ; z]=F\left[\sum_{0}^{\infty} a_{n} t^{n} ; z\right]=\sum_{0}^{\infty} k_{n} a_{n} z^{n}
$$

i cui coefficienti sono i prodotti dei coefficienti corrispondenti delle due serie (18) e (21). Per il teorema generale sulle singolarità, la $f(z)$ potrà essere singolare solo per quei valori $\bar{z}$ in cui sono singolari le funzioni

$$
v\left(z, \beta_{s}\right)=-\frac{1}{\beta_{s}} k\left(\frac{z}{\beta_{s}}\right),
$$


valori che si hanno quando è

$$
\frac{\bar{z}}{\beta_{s}}=\alpha_{r}, \quad \bar{z}=\alpha_{r} \beta_{s} .
$$

Si riottiene così il teorema del Prof. $\mathrm{Hadamard}$ col suo stesso metodo, il quale applicato alla formula integrale (16) $\mathrm{s}^{\mathrm{i}}$ rivela di una portata ben maggiore, fornendo la risoluzione del problema delle singolarità, non pei soli funzionali normali, ma per tutti i funzionali lineari misti.

\section{FUNZIONALI LINEARI MISTI DEL CICLO CHIUSO.}

Oltre i funzionali normali, consideriamo quei funzionali lineari misti, permutabili con l'operazione $S_{\omega}[y(t) ; z]=y(z+\omega)$ che ad ogni funzione $y(t)$ fa corrispondere la funzione $y(z+\omega)$ ( $\omega$ costante qualunque), cioè i funzionali $F$ per cui è identicamente

(24) $\quad F[y(t+\omega) ; z]=F[y(t) ; z+\omega]=f(z+\omega)$

se $f(z)=F[y(t) ; z]$. Segue di qui, derivando rapporto ad $\omega, F\left[y^{\prime}(t+\omega) ; z\right]=f^{\prime}(z+\omega)$, in particolare, per $\omega=o$,

$$
F\left[y^{\prime}(t) ; z\right]=f^{\prime}(z)
$$

mentre, se $y(t)$ è una qualunque funzione periodica con periodo $\omega$, cioè $y(t+\omega)=y(t)$, segue dalla (24) che anche la funzione corrispondente $f(z)$ è pure periodica collo stesso periodo.

Secondo una denominazione del Prof. Volterra, questi funzionali lineari saranno detti perciò funzionali del ciclo chiuso. Essi sono d'importanza fondamentale nella fisica, perchè, quando si ammetta il postulato della costanza delle leggi naturali, segue necessariamente che ogni volta che due grandezze fisiche, funzioni del tempo, sono dipendenti linearmente l'una dall'altra, tale dipendenza lineare è sempre espressa da un funzionale del ciclo chiuso. Questo è il caso, per esempio, della intensità di corrente che attraversa una determinata sezione di un circuito non ferromagnetico, la quale è una funzione del tempo che dipende linearmente dalla forza elettromotrice (funzione arbitraria del tempo); tali dipendenze funzionali (funzionali lineari misti del ciclo chiuso) sono state studiate estesamente dal Prof. Giorgi col nome di "conduttanze funzionali“.
I funzionali del ciclo chiuso sono caratterizzati dal fatto di avere per indicatrice $v(z, \alpha)$ una funzione del tipo

$$
v(z, \alpha)=h(z-\alpha)
$$

cioè una funzione della differenza del parametro e dell'indice. Essi (come del resto i funzionali normali) sono tutti permutabili tra loro. Se sono $\alpha_{r}$ i punti singolari della funzione $h(z), \beta_{s}^{2}$ quelli della funzione $y(t)$, a cui si applica il funzionale del ciclo chiuso che ha l'indicatrice (26) la funzione $f(z)$ corrispondente

$$
f(z)=F[y(t) ; z]=h(z-t) y(t)
$$

avrà i suoi punti singolari tra i valori $\bar{z}$, per cui è

(teorema di Hurwitz).

$$
\bar{z}-\beta_{s}=\alpha_{r .} \quad \bar{z}=\alpha_{r}+\beta_{s}
$$

FUNZIONALI ESPRIMIBILI CON UN NUMERO FINITO DI OPERAZIONI ELEMENTARI.

Indicando, col Prof. Pincherle, come „operazioni elementari" le operazioni di derivazione, sostituzione di una funzione nota al posto della variabile indipendente, moltiplicazione per una funzione nota, si trova poi che condizione necessaria e sufficiente, perchè un funzionale lineare misto $F[y(t) ; z]$, di indicatrice $v(z, \alpha)$, sia esprimibile con un numero finito di operazionielementari è che lindicatrice sia una funzione razionale dell'indice $\alpha$.

I FUNZIONALI ANALITICI NON LINEARI; VARIAZIONE PRIMA E FUNZIONALE DERIVATO.

Passando dai funzionali lineari, finora considerati, ai funzionali analitici più generali, per cui dunque non si suppone più preventivamente la proprietà distributiva rispetto alla somma, il primo problema che si presenta naturale, come per le funzioni, è quello di vedere come si comporta l'incremento $A F$ subito dal funzionale $F[y(t)]$

$$
\Delta F=F[y(t)+\Delta y(t)]-F[y(t)]
$$

in corrispondenza ad un incremento $\Delta y(t)$, dato alla variabile indipendente. Supponendo $\Delta y(t)$ dipendente analiticamente da 
un parametro $\varepsilon$, in modo che la funzione variata $y(t)+\Delta y(t)$ si mantenga però in un intorno $(r, \sigma)$ in cui il funzionale $F$, analitico localmente, è regolare, avremo che anche l'incremento $\Delta F$ risulterà una funzione analitica di $\varepsilon$.

Se $\Delta_{y}(t)$ è infinitesimo con $\varepsilon$, che prenderemo per infinitesimo principale, chiameremo allora variazione prima della funzione $y(t)$, variabile indipendente, e indicheremo con $\delta y(t)$, il termine di primo ordine, rispetto a $\varepsilon$, nello sviluppo di $\Delta y(t)$ in serie di potenze di $\varepsilon$, cioè

$$
\delta y(t)=\varepsilon\left(\frac{\partial \Delta_{U}(t)}{\partial \varepsilon}\right)_{\varepsilon=0}
$$

mentre chiameremo variazione prima del funzionale $F$, e indicheremo con $\delta F$ il termine di primo ordine, pure rispetto ad $\varepsilon$, nello sviluppo dell'incremento $\Delta F$ (che risulta pure infinitesimo), ciòè

$$
\delta F=\varepsilon\left(\frac{\partial \Delta F}{\partial \varepsilon}\right)_{\varepsilon=0}
$$

$\mathrm{Si}$ ha allora la notevole proprietà che la variazione prima $\delta F$ del funzionale $F$ non dipende da tutto l'incremento $\Delta y(t)$ della funzione variabile indipendente, ma soltanto dal suo termine di primo ordine, cioè dalla variazione prima $\delta y(t)$; anzi di più risulta che $\delta F$ è semplicemente un funzionale lineare di questa variazione $\delta y(t)$. Si intravede così l'importanza capitale dei funzionali lineari per tutta la teoria dei funzionali analitici; per la formula generale (8) avremo infatti, indicando con $v(\alpha)$ l'indicatrice di questo funzionale lineare,

$$
\delta F=v(\alpha) \delta y(\stackrel{*}{*})
$$

La variazione prima $\delta F$ del funzionale $F$ è data dunque dal prodotto funzionale di una funzione $v(\alpha)$, (indipendente dallincremento $\Delta y$ ) per la variazione prima $\delta y(\alpha)$ della funzione variabile indipendente. La funzione $v(\alpha)$, che dipende anche dalla funzione iniziale $y$, compie dunque per il funzionale $F$ l'ufficio analogo a quello della derivata per una funzione $f(x)$ (è infatti $d f=f^{\prime}(x) d x$ ); è quindi naturale indicarla col nome di funzionale derivato del funzionale
$F$ nel punto $\alpha$, e col simbolo $F^{\prime}[y(t), \alpha]$. La sua formula di definizione si ha ponendo nella (32)

Si ottiene cosi

$$
\delta_{y}(t)=\Delta y(t)=\frac{\varepsilon}{t-\alpha}
$$

$$
\delta F=\varepsilon v(\alpha)=\varepsilon F^{\prime}[y(t), \alpha]
$$

e confrontando colla (31)

$$
\text { (33) } \quad v(\alpha)=F^{\prime}[y(t), \alpha]=\left(\frac{\partial}{\partial \varepsilon} F\left[y(t)+\frac{\varepsilon}{t-\alpha}\right]\right)_{\varepsilon=0} \text {. }
$$

Il funzionale derivato $F^{\prime}$ è un funzionale misto che dipende ancora, in generale, dalla funzione iniziale $y(t)$, e dall'indice $\alpha$ di derivazione; esso serve a calcolare la variazione prima $\delta F$ mediante il prodotto funzionale di $F^{\prime}$ per $\delta y$. Derivando ancora questo funzionale $F^{\prime}$ in un altro punto $\beta$ si avrà il funzionale derivato secondo $F^{\prime \prime}[y(t), \alpha, \beta]$ di $F$ nei punti $\alpha$ e $\beta$, così di seguito potranno definirsi tutti i successivi funzionali derivati $F^{(n)}\left[y(t), \alpha_{1}, \alpha_{2}, \ldots \alpha_{n}\right]$ per $n$ intero positivo qualunque.

Il funzionale derivato " $n$-esimo è sempre una funzione analitica simmetrica degli $n$ indici di derivazione $\alpha_{1}, \alpha_{2}, \ldots \alpha_{n}$.

Per il calcolo dei funzionali derivati esiste poi un completo sistema di regole, che ricordano molto quelle per le derivate ordinarie, mentre per calcolare la derivata ordinaria della funzione analitica $f(u)=F_{t}[y(t, u)]$ si ha la formula

$$
f^{\prime}(u)=\frac{d}{d u} F_{t}[y(t, u)]=F_{t}^{\prime}[y(t, u), \alpha] \frac{\partial y(\stackrel{*}{\alpha}, u)}{\partial u} .
$$

\section{SVILUPPO DI UN FUNZIONALE ANALITICO IN SERIE DI VOLTERRA.}

In particolare, la formula precedente ci permette di calcolare il valore di un funzionale analitico $F$, regolare in un intorno $(r, \sigma)$ di una funzione $y(t)$, per ogni funzione $y(t)+\varphi(t)$ di questo intorno (tutto l',elemento" di $F$ di centroy), quando se ne conoscano tutti i successivi funzionali derivati per la funzione $y(t)$. Posto infatti $y(t, u)=y(t)+u \varphi(t)$, la funzione $f(u)=$ $=F_{t}[y(t)+u \varphi(t)]$ risulta olomorfa in tutto un cerchio di centro $u=0$ e raggio $>1$. La serie di $\mathrm{Mac}$ Laurin

$$
f(u)=f(0)+\sum_{1}^{\infty} \frac{u^{n}}{n !} f^{(n)}(0)
$$


è quindi assolutamente convergente per $u=1$, e poiché, applicando successivamente la (34) stessa, si trova

$$
f^{(n)}(0)=F^{(n)}\left[y(t), \underset{*}{\alpha_{1},}, \alpha_{*}, \ldots \alpha_{*}\right] \varphi\left(\alpha_{1}^{*}\right) \varphi\left(\alpha_{2}^{*}\right) \ldots \varphi\left(\alpha_{n}^{*}\right)
$$

avremo dalla (35), per $u=1$,

$$
f(1)=F[y(t)+\varphi(t)]=
$$

$$
=F[y(t)]+\sum_{1}^{\infty} \frac{1}{n !} F^{(n)}\left[y(t), \underset{*}{\alpha_{1}}, \alpha_{*}, \ldots \dot{\alpha}_{*}\right] \varphi\left(\alpha_{1}^{*}\right) \varphi\left(\alpha_{*}^{*}\right) \ldots \varphi\left(\alpha_{n}^{*}\right)
$$

Questa formula, che ci fornisce il valore del funzionale analitico $F$, per ogni funzione di un intorno di $y$, in cui $F$ è regolare, mediante una serie, il termine $n$-esimo della quale si calcola eseguendo $n$ prodotti funzionali successivi per l'incremento $\varphi$, fa perfetto riscontro, nella teoria dei funzionali analitici, all'analogo sviluppo di Taylor nella teoria delle funzioni analitiche. In particolare, se il funzionale $F$ è analitico in senso stretto, basterà conoscere tutti i suoi successivi derivati $F^{(n)}$, per una funzione regolare $y(t)$, per avere, colla (36), tutto un elemento di $F$ di centro $y(t)$, e quindi per determinare (con prolungamenti analitici) il valore di $F$ per qualunque altra funzione del suo campo naturale di esistenza.

Lo sviluppo generale (36), che vale per ogni funzionale $F$, analitico localmente, comprende anche, come casi particolari, quello dato dal Prof. Volterra (serie di integrali ordinari) pei funzionali nel campo reale, „regolari“ secondo la sua definizione, e quello più recente del Prof. Fréchet (1910), notevolmente più generale (serie di integrali di Stieltjes); da ciò il nome di serie di Volterra generalizzata, con cui indicheremo lo sviluppo (36).

\section{MASSIMI E MINIMI DEI FUNZIONALI REALI.}

Indicando col nome di funzionale reale un funzionale analitico $F[y(t)]$ che assume valore reale per ogni funzione reale per cui è definito, ci si può proporre il problema di determinare quelle funzioni $y_{0}(t)$, se esistono, nel cui intorno $F$ assume sempre un valore minore di $F\left[y_{0}(t)\right]$ (massimo), o sempre un valore maggiore (minimo).

Per ogni funzione reale $y_{\mathrm{c}}(t)+\varphi(t)$ che appartenga a quell' intorno, la funzione $f(u)=F_{t}\left[y_{o}(t)+u \varphi(t)\right]$, reale per valori reali di u, dovrà pure ammettere rispettivamente un massimo o minimo per $u=0$, e quindi dovrà essere necessariamente

$$
f^{\prime}(o)=F^{\prime}\left[y_{0}(t), \alpha\right] \varphi(\alpha)=0
$$

qualunque sia l'incremento $\varphi$. Dunque l'equazione funzionale a cui debbono necessariamente soddisfare quelle funzioni $y_{o}(t)$, dove $F$ diventa massimo o minimosi ha dalla $(37)$, p er $\varphi(t)=\frac{1}{t-a}$, sotto la forma

$$
F^{\prime}\left[y_{0}(t), \alpha\right]=0 \text {. }
$$

$\mathrm{Si}$ potrebbe vedere che nel caso dei particolarissimi funzionali reali, studiati nell'ordinario calcolo delle variazioni, l'equazione (38) porta effettivamente alla ben nota equazione differenziale di Eulero.

N. B. La teoria dei funzionali analitici si trova esposta in riassunto in una mia memoria, pubblicata nei Rendiconti del Seminario Matematico della R. Università di Roma, anno 1925-26, $e$, per esteso, in una memoria più vasta in corso di stampa per cura della R. Accademia dei Lincei.

La maggior parte dei risultati sono però già stati dati nelle seguenti Note preventive:

„Le funzionali lineari analitiche e le loro singolarità " Rend. Lincei, Vol. $\mathrm{I}^{\circ}, \mathrm{S} .6$, $\mathrm{I}^{\circ}$ sem., 1925.

"La derivazione delle funzionali analitiche" Rend. Lincei, id.

„Le operazioni distributive esprimibili con un numero finito di operazioni elementari“" Boll. dell’ Un. Mat. It., Anno 4, 1925.

„Determinazione dei gruppi a un parametro di funzionali lineari“ Rend. Lincei, Vol. 3, S. 6, I ${ }^{\circ}$ Sem. 1926.

„I funzionali analitici non lineari“ Rend. Lincei; id.

"La polidromia dei funzionali analitici lineari" Rend. Lincei, Vol. $4^{\circ}$, S. 6, $2^{\circ}$ Sem. 1926.

"Les fonctionnelles analytiques qui sont des fonctions d'un nombre fini de fonctionnelles linéaires" C. R. Ac. des Sciences, T. $183,2^{\circ}$ Sem. 1926.

"Sur une classe de fonctionnelles analytiques" C. R., Académie des Sciences, T. 183, $2^{\circ}$ sem. 1926. 
"La teoria dei funzionali analitici nell'integrazione delle equazioni lineari a derivate parziali di qualsiasi ordine" Rend. Lincei, Vol. $4^{\circ}$, S. $6,2^{\circ}$ sem. 1926.

"I funzionali analitici delle funzioni di due variabili complesse" Rend. Lincei, Vol. 5, S. 6, I’ sem. 1927.

(Reçu par la Rédaction le 14. XII. 1928).
Beiträge zur Theorie der schlichten Funktionen von

\section{Z. W. BIRNBAUM (Lwów).}

Eine in einem Bereiche $B$ der komplexen Zahlenebene regulär analytische Funktion $w=f(z)$ heisst schlicht in diesem Bereiche, wenn sie in $B$ jeden Wert höchstens einmal annimmt, d. h. wenn der Bildbereich jeden Punkt der $w$-Ebene höchstens einmal überdeckt.

Der erste, der sich mit den schlichten Funktionen befasste, war Koebe, dem dann Plemelj, Pick, Bieberbach, Faber, Löwner u. a. folgten. Ihre Untersuchungen förderten die Tatsache zu Tage, dass die Forderung der Schlichtheit eine starke Einschränkung für die Funktionen bedeutet. Der erste grundlegende Satz auf diesem Gebiete war der „Verzerrungssatz“ des Herrn $\mathrm{Koebe}^{1}$ ), welcher in seiner endgiltigen, von den Herren Pick ${ }^{2}$ ) und Bieberbach ${ }^{3}$ stammenden Fassung folgendermassen ausgesprochen werden kann:

(I) Wenn $f(z)=z+\sum_{v=2}^{\infty} a_{v} z^{v}$ eine im Einheitskreise $|z|<1$ schlichte Funktion ist, so gilt die folgende Ungleichung:

$$
\frac{1-|z|}{(1+|z|)^{3}} \leqq\left|f^{\prime}(z)\right| \leqq \frac{1+|z|}{(1-|z|)^{3}} \text {. }
$$

1) P. Koebe: Uber die Uniformisierung der algebraischen Kurven durch automorphe Funktionen mit imaginärer Substitutionsgruppe, Gött. Nachr. 1909 p. $68-76$.

58 G. Pick: Über den Koebe'schen Verzerrungssatz, Leipz. Ber. 1916

$\left.{ }^{3}\right)$ L. Bieberbach: Über die Koeffizienten derjenigen Potenzreihen, welche eine schlichte Abbildung des Einheitskreises vermitteln, Sitzber. d. kgl Akad. Berlin 1916, p. 940-955. 